\title{
Risk Perception and Its Foundation among Swedish Individuals Occupationally Exposed to Air Pollutants and Chemicals: A Comparison of 1975 and 2011
}

\author{
Linda Schenk \\ Division of Philosophy, Royal Institute of Technology, Teknikringen 78B, 10044 Stockholm, Sweden \\ Correspondence should be addressed to Linda Schenk; schenk@kth.se
}

Received 13 May 2013; Accepted 10 July 2013

Academic Editors: M. F. Allam, S. M. Pezzotto, P. Plans, I. Szadkowska-Stanczyk, and G. Tolomiczenko

Copyright @ 2013 Linda Schenk. This is an open access article distributed under the Creative Commons Attribution License, which permits unrestricted use, distribution, and reproduction in any medium, provided the original work is properly cited.

\begin{abstract}
Although work environment conditions have improved much in the last decades, occupational exposures to chemicals and air pollutants still cause adverse health effects. The risk perception of occupational exposures to hazardous substances and the motivations for it have been investigated through a questionnaire study. These results have also been compared to previous studies performed by the largest trade union confederation in Sweden in 1975. In the current survey, 30\% of the respondents believe to be at risk, $48 \%$ not, and $22 \%$ state to be unsure. The proportion of persons experiencing to be at risk due to exposures to chemicals or air pollution at work is unexpectedly similar to that of 1975 . The explanations offered for the risk perception were in many cases vague and of a subjective nature, but again results are similar to those of 1975. References to more solid justifications such as performed exposure measurements or medical health checks are scarce, with the latter case being as scarce today as in 1975 . There are only a few indications that a more systematic management of work environment issues has developed at Swedish workplaces; for instance, managers were more commonly stated as a source of information in 2011 than in 1975.
\end{abstract}

\section{Introduction}

Working conditions have a major impact on public health and welfare. In Sweden, occupational diseases are estimated to cause at least 1000 fatalities per year [1]. Although the work environment in Sweden has gone through major improvements from a physicochemical perspective during the past forty years [2], exposure to hazardous air pollution should not be considered an issue of the past. Statistics are available on the percentage of employed Swedes stating to be exposed to air pollutants, defined as palpable dust or chemicals, during at least $1 / 4$ th of their time at work since 1997. The percentages are quite stable; in $1997,23 \%$ of the workforce stated to be exposed at least $1 / 4$ th of their time at work [3]; in 2011, the corresponding number was $22 \%$ [4]. The highest level during this period, 24\%, was recorded in 2007 [5]. As there are approximately 4.5 million workers in Sweden, this can be expressed as close to one million Swedes are exposed to air pollutants at work for at least a $1 / 4$ th of their working time. A survney performed in spring 2010 indicated that
$0.5 \%$ of the employed women and $0.4 \%$ of the employed men had experienced work-related disorders due to chemical exposures during the last twelve months [6].

Individuals' risk perception and the foundations for that belief are important targets for successful risk management. A major component in the work towards a safer work environment has been the technical developments that have decreased the exposures to physical as well as chemical risk factors [2]. Processes in large chemical industries are mostly closed today. Exposures can thus be argued to mainly occur in smaller workplaces where closing of the processes is not technically or economically feasible. Such exposures can in many cases be reduced or even eliminated by the use of proper personal protective equipment. This, however, places a large degree of responsibility of the occupational hygiene on the individual employees to take the time and effort to actually use the protective equipment and to use it correctly. Studies have shown that individuals' safety behaviour is affected by the perception of risk; for example, individuals who perceive themselves to be at risk are more prone to using 
protective equipment [7]. Investigating the risk perception and its foundation might thus give insights that can be used to target individuals' safety behaviour.

Previous questionnaire studies performed by the Swedish Trade Union Confederation (LO) provide a documentation of the development of the Swedish work environment during the 1970 s $[8,9]$. The LO is a nationwide confederation of, currently 14 , trade unions in different areas of industry, and the trade union confederation target the largest group of manual workers in Sweden. In 1968 to 1969 the LO sent out the first questionnaire to its members in order to get a picture of the physical issues in the work environment. This LO survey aimed at investigating if and to what degree the members were concerned about such issues and if they were experiencing adverse health effects due to these [10]. In the mid-1970s, partly due to this first LO survey [2,9], awareness of chemical issues increased, and, in 1974 to 1975, a new LO survey was performed targeting the chemical health risks [8]. This second survey will henceforth be referred to as the 1975 LO survey. In 1980 once again, an LO survey was sent out, this time designed so as to make the results comparable mainly with the 1975 LO survey, but also with the first LO survey [9]. In addition to workers in general, the 1980 LO survey also targeted the group of safety representatives (although the legal responsibility for a safe working environment lies on the employer, in Sweden, the safety representatives potentially play an important role for risk management at the workplace. Safety representatives are appointed by the employees, most commonly through a union. Being a safety representative is thus a commission of trust to represent the employees in questions concerning health and safety at work) with an extended survey.

Since the 1970s the emphasis on the employers' responsibility for ensuring a safe workplace and also for informing their employees about workplace risks has increased in Sweden. The Swedish Work Environment Act was issued in 1977, with the aim to prevent ill health and accidents resulting from working conditions. The act also places the main burden of responsibility for this task on the employer [11]. In the 1990s legal requirements for the employer to work systematically with fulfilling the requirements of the Work Environment Act which were in the early 2000s developed to the Provisions on Systematic Work Environment Management (SWEM) [12], issued by the Swedish Work Environment Authority under the Work Environment Act. According to the SWEM provisions, managers and supervisors are required to be aware of the relevant regulations and how these should be applied in the operations. These provisions also state that the employer is responsible for giving the employees the information necessary for preventing ill health and accidents at work. The requirements forinforming the employees about workplace risks are also highlighted in the Swedish Work Environment Authority's provisions specifically focused on chemicals safety in the work environment [13], with the addition that the employer also should ensure that the employee has understood the information. Work environment encompasses all aspects of wellbeing at work and is not limited to the health effects of chemicals and dust. This paper is, however, limited to these aspects of work environment.
A regulatory tool specifically aimed at airborne chemicals and dust in the workplace is to set a list of occupational exposure limits (OELs). The first Swedish list of OELs was issued in 1969. This introduction of OELs is seen as a tool of further identifying and specifying the employers' responsibility for a safe workplace. Swedish OELs are a numerical limit of the highest allowable concentration of an air pollutant in the breathing air of workers [14]. Since the first list was issued it has been updated at regular intervals, increasing the number of substances that are covered from 75 in 1969 [15] to the current number of around 400 [14].

The overall developments in the Swedish regulation of work environment risks, both in general and specifically for air pollutants and chemicals, thus suggest that work environment conditions should have improved and that information regarding occupational risk factors should be provided to employees by their employer. The purpose of the present study is to provide an overview of risk perception of occupational exposure to hazardous substances and the motivations for these perceptions at current Swedish workplaces and to compare this to the results of the previous 1975 LO survey. The results will be discussed from the perspective of the regulatory developments in Sweden that have implications for chemicals safety management at the workplace.

\section{Materials and Methods}

The present study had two different target groups: first, workers that are exposed to air pollutants or chemicals, and, secondly, persons working with occupational health and safety or who are in a management position at a company at which workers are exposed to air pollutants or chemicals. The groups were identified according to the respondents' own perception of their workplace exposure, and no data was collected on specific occupations. In order to reach these groups a questionnaire was sent out to persons above the age of 19 in a nationally representative web-based panel using Sifo Research International. The respondents were first given a series of qualifying questions, and answers from 1017 persons who were within the target groups were collected out of the 3242 respondents who had opened the questionnaire. Seven respondents declined to fill in the questionnaire after reading the introductory information of the questionnaire, and 1737 were screened out due to unemployment or perceived nonexposure. The remaining 481 respondents started the questionnaire but did not finish it before it was closed. These respondents' reasons for not finishing the questionnaire were not further investigated.

A description of the sample is provided in Table 1 . In addition the respondents were asked to indicate their position in their company and the size of their workplace. Of the 1017 respondents, 97 state that they are site managers or owners of the company, 168 state that they are from middle management, 36 state that they work with occupational health and safety issues at their workplace (henceforth called OHS staff), and 79 state that they are safety representatives. The remaining respondents, 637 persons, did not state to have any of these positions and will be referred to as workers. Owners, 
TABLE 1: Position of respondents and size of company where employed.

\begin{tabular}{|c|c|c|c|c|c|}
\hline Number of employees & $\begin{array}{c}\text { Workers } \\
(n=637)\end{array}$ & $\begin{array}{l}\text { Safety representatives } \\
\qquad(n=79)\end{array}$ & $\begin{array}{l}\text { OHS employee } \\
\quad(n=36)\end{array}$ & $\begin{array}{c}\text { Management } \\
(n=265)\end{array}$ & $\begin{array}{l}\text { All respondents } \\
\quad(n=1017) \\
\end{array}$ \\
\hline $1-25$ & $31 \%$ & $25 \%$ & $25 \%$ & $47 \%$ & $35 \%$ \\
\hline $26-50$ & $18 \%$ & $23 \%$ & $25 \%$ & $14 \%$ & $18 \%$ \\
\hline $51-150$ & $20 \%$ & $23 \%$ & $25 \%$ & $19 \%$ & $17 \%$ \\
\hline $151-500$ & $15 \%$ & $14 \%$ & $6 \%$ & $8 \%$ & $13 \%$ \\
\hline $501-1500$ & $8 \%$ & $10 \%$ & $8 \%$ & $7 \%$ & $8 \%$ \\
\hline More than 1500 & $9 \%$ & $5 \%$ & $11 \%$ & $6 \%$ & $8 \%$ \\
\hline Total & $63 \%$ & $8 \%$ & $4 \%$ & $26 \%$ & $100 \%$ \\
\hline
\end{tabular}

site managers, and middle management have been compiled into a management group $(n=265)$.

In order to facilitate a comparison with previous LO surveys, ten items were used from these $[8,9]$. Permission to use these items was sought and obtained from the central administration of LO, as the authors of the survey reports are no longer active. A few of the items were adapted in order to better suit the current information situation, such as the widespread access and use of the Internet. It should be noted that the LO surveys did have a less general selection of respondents than the present study. For instance persons in a higher managerial position commonly belong to a different trade union confederation than LO, since LO mainly targets manual labourers and not academics or "white-collar" workers (http://www.lo.se, 2012-04-26).

These items concerned which health risks posed by air pollution and chemicals at the workplace have been identified by the respondents and also how.

Statistical analyses have been performed in the form of Chi-square tests when the nature of the obtained data allows it. Questions for which multiple answers were possible are not analysed this way, as these would result in imbalanced contingency tables since some respondents gave more responses than others.

\section{Results and Discussion}

In Section 3.1 to 3.4 results from the present study regarding risk perception, motivations and work environment are presented and compared to previous findings from the LO surveys of 1975 and, when applicable, from 1980.

3.1. Perceived Exposure and Occupational Risks. In the present study $46 \%(n=1017+481)$ of those opening the questionnaire $(n=3242)$ experience occupational exposure to air pollutants or chemicals. As not all of these have fulfilled the questionnaire, the results will be based on the 1017 respondents that have given full responses. Approximately one third (30\%) are of the opinion that these exposures might be harmful to their health, while almost half (48\%) of the respondents do not think there is a risk for their health, and the rest (22\%) stated to be unsure.

3.1.1. Perceived Exposure and Occupational Risks: Comparison to 1975. The degree of perceived exposure has lessened somewhat during the last 35 years; in the LO survey of 1975, $54 \%$ of the respondents stated to be exposed to any kind of air pollutants; in the present study the corresponding figure could be deduced to $46 \%$. This is higher than the corresponding figures from the Swedish statistics for 2011 [4] as the respondents to the present survey, in line with the LO survey of 1975 , were not limited to those exposed during at least $1 / 4$ th of their working time.

The degree of perceiving oneself at risk due to these exposures has decreased more between 1975 and 2011 than the perceived exposure. In 1975, 43\% of the respondents found the exposures to pose a risk to their health and $57 \%$ did not. In the present study, $48 \%$ of respondents stating to be exposed did not perceive these exposures to pose a risk to their health. In this sense, the perception of this aspect of workplace safety seems to have deteriorated rather than improved in the past 35 years. However, there were differences in response options regarding this question in the two surveys. In the present study respondents were also given the option "unsure" which respondents to the LO survey were not. Individuals that would have selected "unsure" in the 1975 survey were hence forced to choose either risk or no risk, and it might be more plausible to respond that there is no risk in the case of uncertainty. It may also be that the level of what is perceived adverse or not when it comes to health effects has changed; that is, the workforce was more accepting in the 1970s than presently.

3.2. Foundation of Risk Perception. Among the respondents that have reported that they find their workplace exposure to pose a risk to their health, $16 \%$ have also specified that they have experienced adverse health effects (49 out of 308 ). If we assume that respondents that report that they do not find their work environment to pose a risk to their health also have not experienced any adverse health effects, we can conclude that less than $4.8 \%$ of the target group has experienced adverse effects due to exposure to chemicals or air pollutants.

The respondents were asked the question "Have you received information that your work environment might pose a health risk, and $28 \%$ claimed" to not have been given such information while $72 \%$ have and also indicated by which sources such information has been given. This means that less than half (42\%) of the persons having received information that their work environment might pose a health risk believe that there is a risk for them. Also, 25\% $(n=252)$ of 


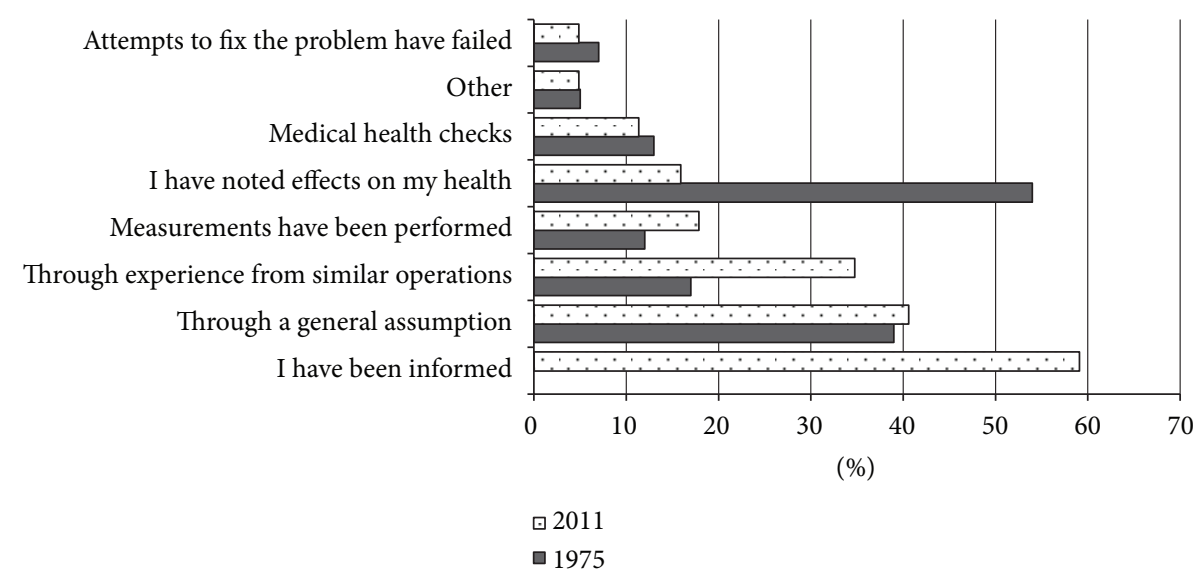

FIGURE 1: Reasons for thinking that there is a health risk. Multiple answers possible.

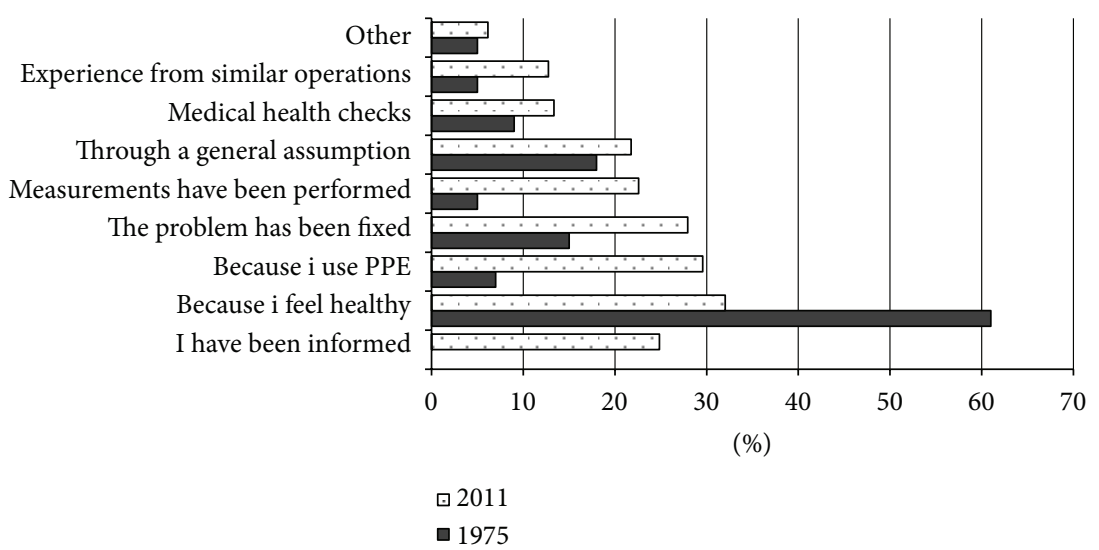

FIGURE 2: Reasons for not believing that there is any health risk. Multiple answers possible. PPE: personal protective equipment.

the respondents state to not have received any information of either there being a risk or not being a risk; $75 \%$ of these are workers and 39\% work in companies with 25 or less employees. Nevertheless, received information is the most commonly stated foundation for the perceived risk (Figure 1). Of the respondents that believe their work environment poses a risk to their health, 59\% (182 out of 308) state that they reached this conclusion partly or fully by receiving information that there might be a health risk (Figure 1). Experience from similar operations and "a general assumption" are the second most commonly stated foundations given by somewhat more than $10 \%$ of the respondents (Figure 1). Justifications based on objective sources of information such as performed measurements and medical health checks are relatively scarce as the foundation for both perceiving a risk and perceiving no risk (Figures 1 and 2). Combined, 29\% of the respondents refer either to both medical health checks and technical measurements or one of these, as a reason for perceiving a risk. A larger share, $42 \%$, justifies not feeling at risk by referring to technical measurements or medical health checks.

3.2.1. Foundation of Risk Perception: Comparison to 1975. In the present study $4.8 \%$ of the respondents report to have experienced adverse health effects due to exposure to chemicals or air pollution. This is less than a fourth of the corresponding number from the LO survey of 1970 . The selection of respondents might, however, present an overrepresentation of these kinds of effects among the general Swedish workforce as persons perceiving themselves as exposed to chemicals and/or air pollutants were specifically targeted.

Comparing the foundation for the risk perception the most apparent difference between the results from the present study and those of the LO in 1975 is that of respondents having noted effects on their health (Figure 1). Also "feeling healthy" was more commonly stated in 1975 as a reason for not perceiving any risk to one's health from workplace exposures. More objective reasons for thinking there is a risk, such as knowledge of medical health checks or performed exposure assessments, were in 2011 stated by less than $20 \%$, respectively. Again these numbers are very similar to those of the 1975 LO survey. When it comes to the foundation for perceiving no risk, more objective reasons are more common in the present study than in 1975 (Figure 2). In addition, stating that "the problem has been fixed" was a more common answer to why respondents do not perceive themselves at risk in 2011 than in 1975. However, subjective and vague reasons such as "a general assumption" or "feeling healthy" 
TABLE 2: Have risks to health or other troublesome conditions been measured by technical means at your workplace?

\begin{tabular}{lcccccc}
\hline & & \multicolumn{5}{c}{ Number of employees where respondents work } \\
& $1-25$ & $26-50$ & $51-150$ & $151-500$ & $501-1500$ & $>1500$ \\
\hline Yes & $14 \%(49)$ & $25 \%(44)$ & $32 \%(64)$ & $34 \%(44)$ & $42 \%(32)$ & $44 \%(37)$ \\
No & $65 \%(228)$ & $39 \%(70)$ & $37 \%(74)$ & $32 \%(41)$ & $29 \%(22)$ & $31 \%(26)$ \\
Unsure & $21 \%(74)$ & $30 \%(64)$ & $30 \%(60)$ & $34 \%(43)$ & $20 \%(23)$ & $26 \%(22)$ \\
\hline
\end{tabular}

$\chi_{(\mathrm{df}=5, n=731)}^{2}=81.89, P=0.000$, excluding unsure respondents.

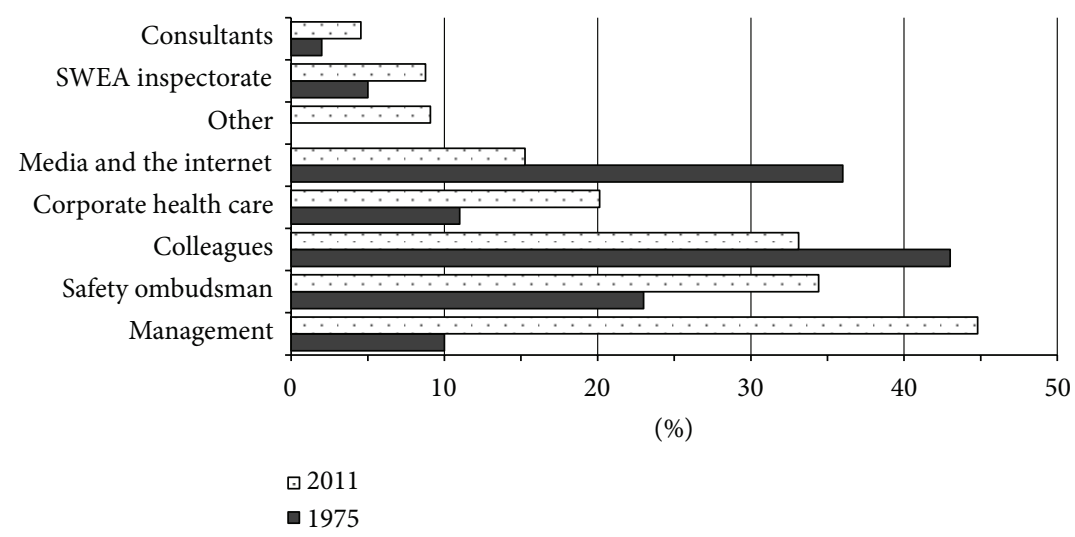

FIGURE 3: Sources of information about occupational health risks stated by respondents that also have stated that they consider their work environment to pose a health risk $(n=308)$. In addition, in 2011, $15 \%$ stated to have no sources. Multiple answers possible. SWEA: Swedish Work Environment Authority.

are still given by a noteworthy proportion of the respondents. It should be noted that believing to not be at risk because one feels healthy disregards the potential for long-term effects, which most often also are the most severe effects.

In the 1975 LO survey only those stating that they perceived themselves to be at risk were asked about a source of information. Among these the most common source was stated to be work colleagues. Television and papers were also stated to be important sources of information. In Figure 3 the sources of information for respondents stating that they perceive their work environment to pose a risk for their health in the present study are compared to the corresponding numbers from the 1975 LO survey. The influence of media seems to have declined considerably, even including the Internet, as has to some degree that of work colleagues. Management and safety representatives were more commonly stated to be sources of information in 2011 than in 1975.

3.3. Exposure Assessment. Among the respondents of the present study $27 \%$ report that technical measurements have been performed at their workplace, $45 \%$ report that no measurements have been performed, and $28 \%$ were unsure (Table 2). Of the respondents reporting that technical measurements had been performed, 43\% (34 out of 79) were safety representatives. Technical measurements, or awareness of these, are relatively more common in medium sized and large enterprises than in small ones $\left(\chi_{(\mathrm{df}=5, n=731)}^{2}=81.89\right.$, $P=0.000$, excluding unsure respondents). The respondents were also asked what was measured (Table 3 ) and by whom (Figure 4) ; 73\% reported that measurements concerned air quality.
TABLE 3: What was measured? Several answers possible.

\begin{tabular}{lc}
\hline Air quality for dust and smoke $^{\mathrm{a}}$ & $60 \%(118)$ \\
Air quality for chemicals $^{\mathrm{a}}$ & $56 \%(111)$ \\
Air quality for exhaust fumes & $\mathrm{a}$ \\
Ergonomic issues & $29 \%(57)$ \\
Other physical issues (climate) & $66 \%(130)$ \\
Unsure & $70 \%(139)$ \\
\hline
\end{tabular}

${ }^{\mathrm{a}}$ In total, $73 \%(n=198)$ of the answers concerned one or several of these categories.

3.3.1. Exposure Assessments: Comparison to 1975. A difference between the LO surveys and the present study concerning technical measurements is that the LO surveys only gave this question to safety representatives. In $1975,52 \%$ of the safety representatives stated that technical exposure assessments had been performed at their workplace. In the present study $43 \%$ of the safety representatives reported to be aware of technical measurements, while for all respondents the corresponding number was $27 \%$. Due to the small number of safety representatives in the present study, it is difficult to say for certain that the frequency of exposure measurements was lower in 2011 than in 1975. In the 1980 LO survey, air pollution had been investigated at the workplace according to $43 \%$ of the safety representatives, and $75 \%$ of these involved technical measurements.

In the present study it was found that technical measurements have been more frequently performed in medium and large companies than in small ones. This finding is in concordance with the findings from the LO surveys from 1975 


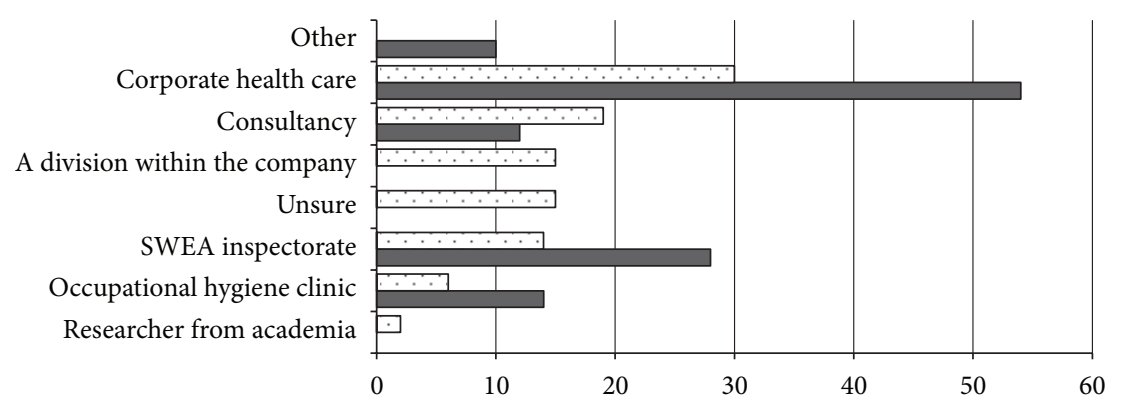

(\%)

$\square 2011$

$\square 1975$

FIGURE 4: Respondents' answer to who performed the last (if several) technical measurements at their workplace. The alternative "other" from 1975 includes academia and a division within the company. SWEA: Swedish Work Environment Authority.

and 1980. A possible interpretation of this trend is that smaller companies are affected to a lesser extent by occupational risk factors. However, this is implausible, as previous studies have indicated that small and medium sized enterprises do not invest much resources in improving occupational hygiene [16]. Rather it is judged as more probable that awareness of occupational risk factors is higher among staff of larger companies and/or resources are more restricted in small companies, and thus occupational hygiene issues are not prioritized in these. Needless to say, it is important that also small and medium sized enterprises take preventive actions as about $67 \%$ of the workforce in the EU is employed by small and medium sized enterprises [17].

A comparison of respondents' answers regarding who performed the measurements indicates that the role of occupational hygiene clinics as well as corporate health care has diminished (Figure 4), as has the frequency of measurements performed by the inspectorate under the Swedish Work Environment Authority.

3.4. Identified Issues and Cause of Awareness. Managers, OHS staff, and safety representatives were asked to state what, if any, issues regarding air pollution or handling of chemicals they have spent the most effort on overcoming. The answers written by the respondents have been sorted by the author into larger categories which are given in Table 4 . It was also possible for the respondents to state nothing, which $65 \%$ (248 out of 380) did. Of the 132 respondents that did write one or several issues, $34 \%$ (45) were safety representatives. The percentage of respondents writing a reply to this question was $29 \%$ for companies with 25 or fewer employees and around $39 \%(36-40 \%)$ for the other categories $\left(\chi_{(\mathrm{df}=1, n=380)}^{2}=4.34\right.$, $P=0.037$, comparing companies having 25 or less employees with all larger companies combined).

Among the issues mentioned in Table 4, exposure to dust and fibres and ensuring that hazardous substances are handled in a proper manner as well as issues with ventilation are the most common. Notable is that, although the question asked about which issues regard air pollutants and chemicals, the most common answer was an unspecific statement such as
TABLE 4: Issues regarding air pollution or handling of chemicals.

\begin{tabular}{lc}
\hline Categories & $n$ \\
\hline Chemicals, not otherwise specified & 23 \\
Substitution of hazardous substances & 5 \\
Antibiotics/disinfectants & 3 \\
Solvents & 3 \\
Pesticides/biocides & 2 \\
Fuel & 2 \\
Dust and fibres & 19 \\
Asbestos & 4 \\
Proper handling of hazardous substances & 15 \\
Use of protective equipment & 11 \\
Establish a chemicals registry & 3 \\
Labelling of chemicals and MSDS & 4 \\
To protect myself & 1 \\
Air quality, not otherwise specified & 10 \\
Ventilation & 13 \\
Exhaust fumes & 7 \\
Smoke & 8 \\
Carbon dioxide & 3 \\
Aerosols & 2 \\
Physical factors & 9 \\
Biological factors & 3 \\
\hline
\end{tabular}

This was an open-ended question; 132 out of 380 respondents replied to this question, of which 45 were safety representatives.

MSDS: material safety data sheets.

"chemicals" or "hazardous substances" (17\%); several answers also referred to issues with climate, noise, and ergonomics.

The respondents were also asked how these issues were brought to their attention, and their answers are displayed in Figure 5. The most common answer was "due to experience from similar operations;" however, separating the respondents depending on position, it seems that safety representatives most often get aware of issues due to complaints from staff. These complaints from staff are a relatively more common cause of awareness among safety representatives 


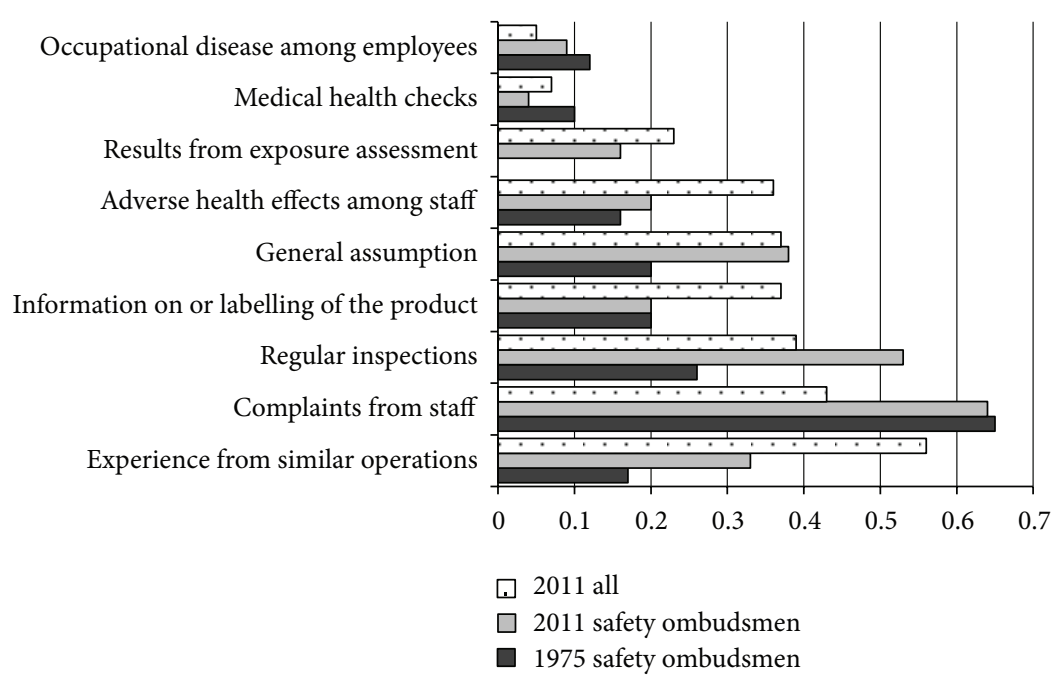

Figure 5: How the identified issues have been brought to the respondents' attention. Multiple answers possible.

than managers and OHS staff, which could be an indication that safety representatives are more trusted by employees and thus are more frequently spoken to about issues concerning the work environment.

\subsubsection{Identified Issues and Cause of Awareness: Comparison} to 1975. Concerning the relationship between number of respondents identifying issues and company size, a similar trend was apparent in the 1975 LO survey as in the present study. In the 1975 LO survey, the largest share of respondents stating not to have any issues with chemicals in the work environment worked at companies with 25 or fewer employees. Similarly, the percentage of informants specifying an issue in the present survey was higher from respondents working at companies larger than 25 employees compared to smaller ones. In the 1975 LO survey dust was the most common issue (34\%), followed by solvents (27\%). In the present study only three of the informants mentioned solvents, and although dust was among the more commonly specified issues only 19 informants mentioned dust or fibres and an additional four mentioned asbestos.

Although it could be expected that requirements for product labelling have been increased since 1975, resulting in an increase of the amount of information available, it seems that the importance of labelling in the identification of occupational hazards has not increased among safety representatives. However, this survey also includes other positions in the company, and among these the labelling seems to be an important source (Figure 5). Referring to "previous experiences from similar operations" or making "a general assumption" is more common among the respondents of the present study than among the safety representatives of the 1975 LO survey. Regular workplace inspections also seem to be more important as raisers of awareness today than in the LO survey of 1975. As would be expected from the 35 years in which work environment could have been improved, referring to occupational illness or results from medical checks is less common in 2011 than in 1975 (Figure 5).

\section{Conclusions}

The differences in perceived exposure and perceived risk are not very large between 1975 and 2011. However, these similarities might be due to differences in perception rather than similarities or work environment conditions. The results presented herein are based solely on what respondents have reported, and true work environment conditions are unknown. Overall, the respondents' answers seem to indicate that work environment conditions have improved since 1975. It was less common in 2011 than in 1975 to refer to occupational diseases, complaints from staff, and medical health checks for identifying a work environment issue. This decrease could be interpreted as preventive work has become increasingly important in comparison to the reactive work. In addition, stating that "the problem has been fixed" was a more common answer to why respondents do not perceive themselves to be at risk in 2011 than in 1975. The present study also elicited fewer responses regarding issues with chemical exposures at the workplace than the 1975 LO survey. Fewer responses regarding identified issues could of course depend on decreased awareness as well as improved work environment conditions. However, also other overviews have concluded that workplace conditions from the perspective of physical and chemical hazards have been improved in Sweden since the 1970s [2].

There are some indications in the collected material that the provisions on SWEM have had an impact. A systematic management of work environment issues requires regular inspections of the workplace. The safety representatives of the present survey more often state that issues with air pollutants and chemicals are identified through such regular inspections than the safety representatives of the 1975 LO-survey. Also, exposure measurements as a reason for not believing there is a risk to one's health were much more commonly stated in 2011 than in 1975. The number of exposure measurements can be expected to have increased as a result of there being many more OELs in force today, but also because the requirements 
to perform workplace risk assessments and to substantiate safety have been increased. An important aspect of SWEM is to inform the employees. It seems that information pathways have improved since 1975, in the sense that management is seen as the major provider of information about workplace risks today. However, although information pathways have improved the results from 2011, it could still be questioned if managers are sufficiently active as information sources. Not even half of the respondents perceiving to be at risk state that management is a source of information about occupational health risks. In addition, the respondents' justifications for their risk perception, or identification of health issues by responsible staff, were in many cases of a subjective and vague nature. References to solid justifications such as technical measurements and medical health checks were scarce. These proportions are strikingly similar to the results of the 1975 LO survey. Also, about one fifth of the respondents in the present study stated to be unsure regarding the risk from their workplace exposures. The obscure justifications and uncertainties might be caused by lacking or ineffective communication about workplace risks. Additional improvements of occupational health may be gained by more stringent enforcement of the SWEM provisions.

\section{Conflict of Interests}

The research was funded by AFA Insurance. The author's affiliation is stated on the title page, and there is no conflict of interests.

\section{Acknowledgments}

The author is grateful to Professor Sven Ove Hansson and Associate Professor Misse Wester, both at the Royal Institute of Technology, for valuable input to the questionnaire design.

\section{References}

[1] B. Järvholm, "Arbetsrelaterade dödsfall i Sverige [Work-related fatalities in Sweden]," Swedish Work Environment Authority, 2010:3, 2010.

[2] K. Frick, "The regulation of systematic work environment management in Sweden-higher ambitions in a weaker Swedish work environment system," in RegulatIng Workplace Risks, pp. 115-140, Edward Elgar Publishing, 2011.

[3] Arbetarskyddsstyrelsen and Statistics Sweden, "Arbetsmiljön 1999 [The Work Environment 1999] Statistiska meddelanden AM 68 SM 0001".

[4] Swedish Work Environment Authority, "Arbetsmiljön 2011 [The Work Environment 2011] Report 2012:4”.

[5] Swedish Work Environment Authority, “Arbetsmiljön 2007 [The Work Environment 2007] Work environment statistics Report 2008:4".

[6] Swedish Work Environment Authority, "Work-realted disorders 2010," Swedish Work Environment Authority, 2010:4, 2010.

[7] P. M. Arezes and A. S. Miguel, "Risk perception and safety behaviour: A study in an occupational environment," Safety Science, vol. 46, no. 6, pp. 900-907, 2008.
[8] E. Bolinder, A. Englund, and E. Magnusson, "Kemiska hälsorisker i arbetsmiljön: en analys baserad på enkätundersökningar bland LO-medlemmar och skyddsombud," Stockholm: Prisma i samarbete med Landsorganisationen i Sverige, 1976.

[9] E. Bolinder and Landsorganisationen i Sverige, "Vad händer med arbetsmiljön?: rapport om LO-medlemmarnas och skyddsombudens erfarenheter," Stockholm: Landsorganisationen: Tiden, 1981.

[10] E. Bolinder, E. Magnusson, and L. Nyrén, "Risker i jobbet: LOenkäten: LO-medlemmarnas uppfattning om arbetsplatsens hälsorisker. [Stockholm]: Prisma i samarb.med Landsorganisationen i Sverige," 1971.

[11] Swedish Parliament, "Swedish Work Environment Act," 1977.

[12] Swedish Work Environment Authority, "Systematiskt Arbetsmiljöarbete [Systematic Work Environment Management]," 2001.

[13] Swedish Work Environment Authority, "Kemiska Arbetsmiljörisker [Chemical Risks at the Workplace]," AFS 2011:19. Stockholm: Elanders, 2011.

[14] Swedish Work Environment Authority, "Hygieniska gränsvärden [Occupational Exposure Limits]," 2011.

[15] S. O. Hansson, Setting the Limit: Occupational Health Standards and the Limits of Science, Oxford University Press, New York, NY, USA, 1998.

[16] P. Stephens and Great Britain. Health and Safety Executive., and Vectra Group Limited., Occupational Health and SMEs: Focused Intervention Strategies: Part 1-Overview Report: Part 2-Substantiating Chapters, HSE Books, Sudbury, Canada, 2004.

[17] P. Wymenga, V. Spanikova, J. Derbyshire, and A. Barker, "Are EU SMEs recovering? Annual Report on EU SMEs 2010/2011," European Commission, DG-Enterprise, 2011. 


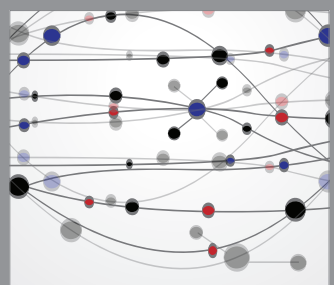

The Scientific World Journal
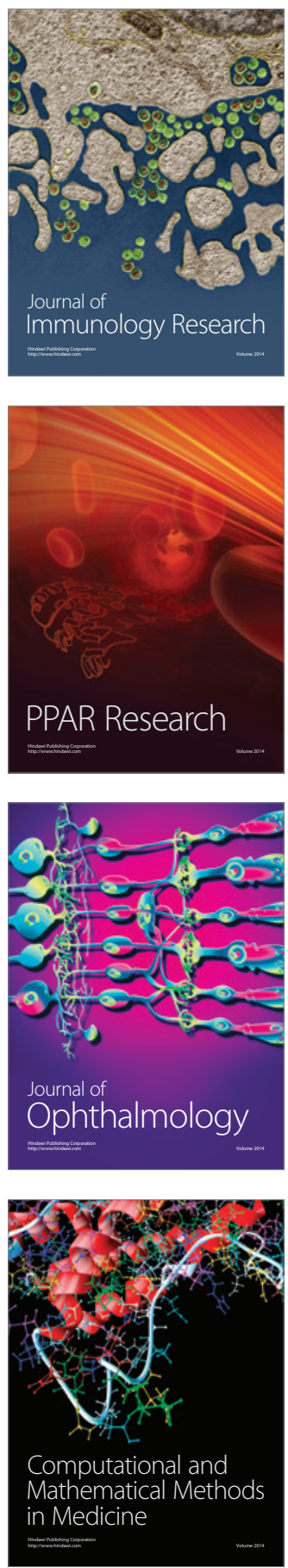

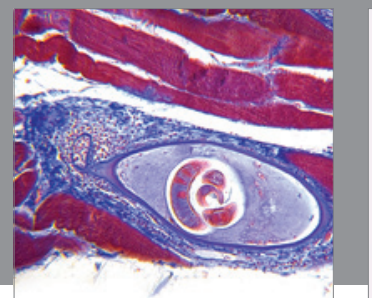

Gastroenterology

Research and Practice
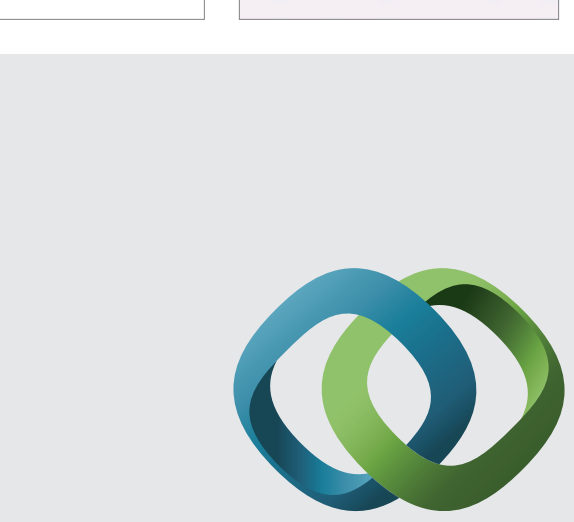

\section{Hindawi}

Submit your manuscripts at

http://www.hindawi.com
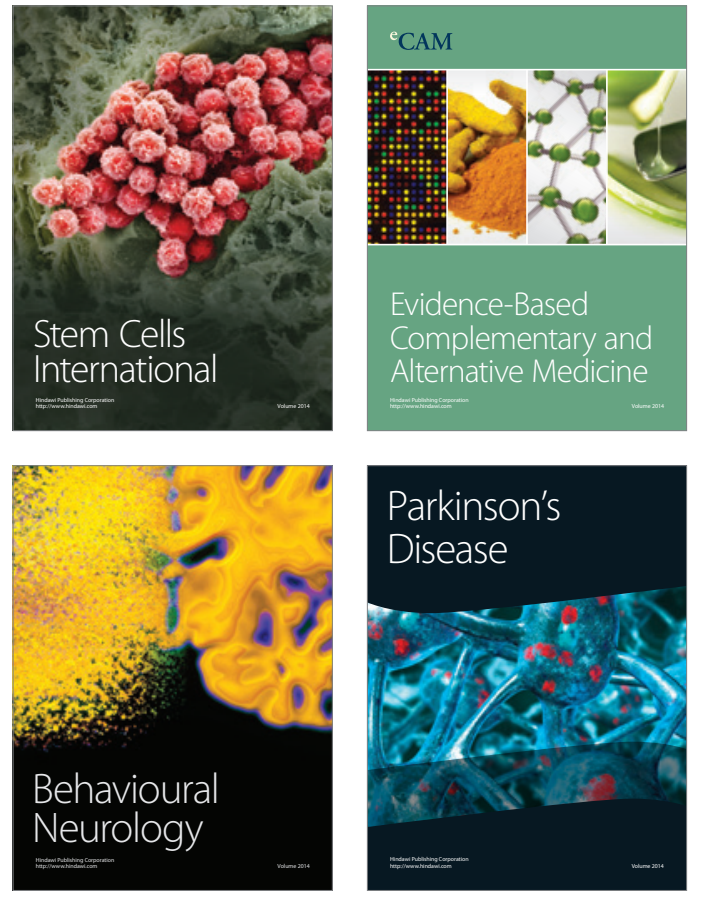
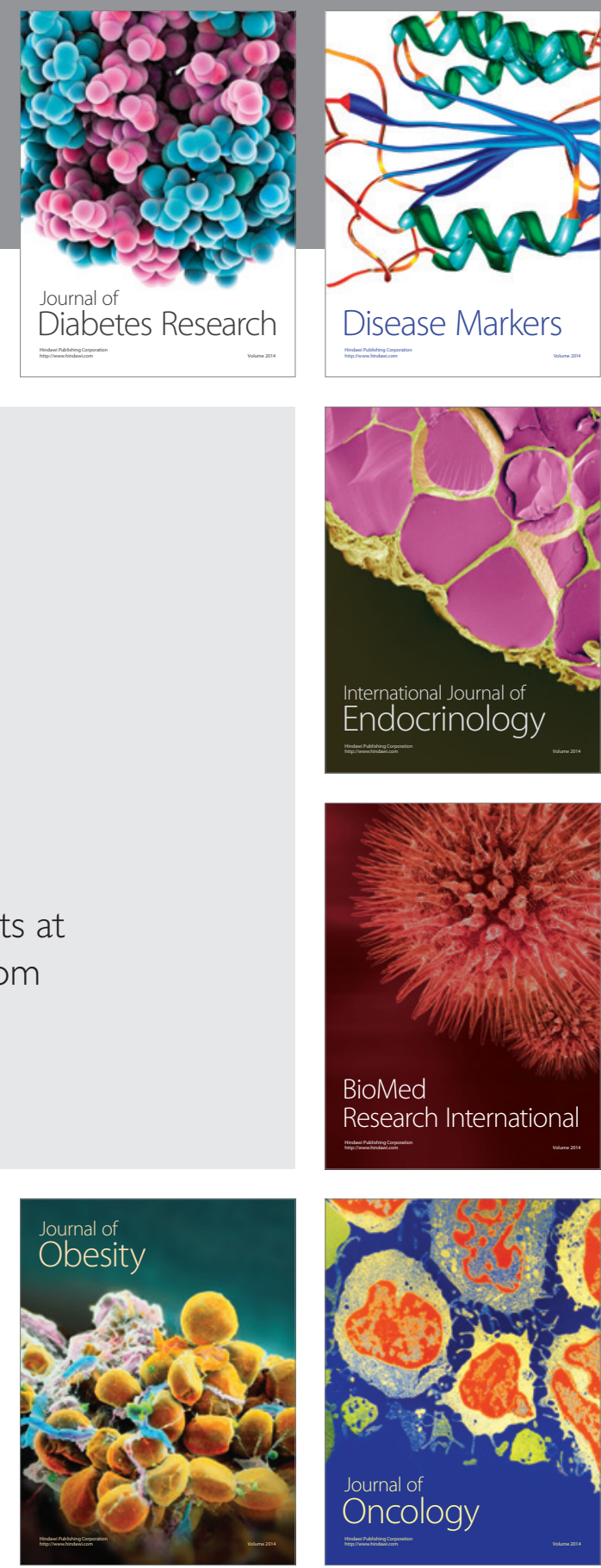

Disease Markers
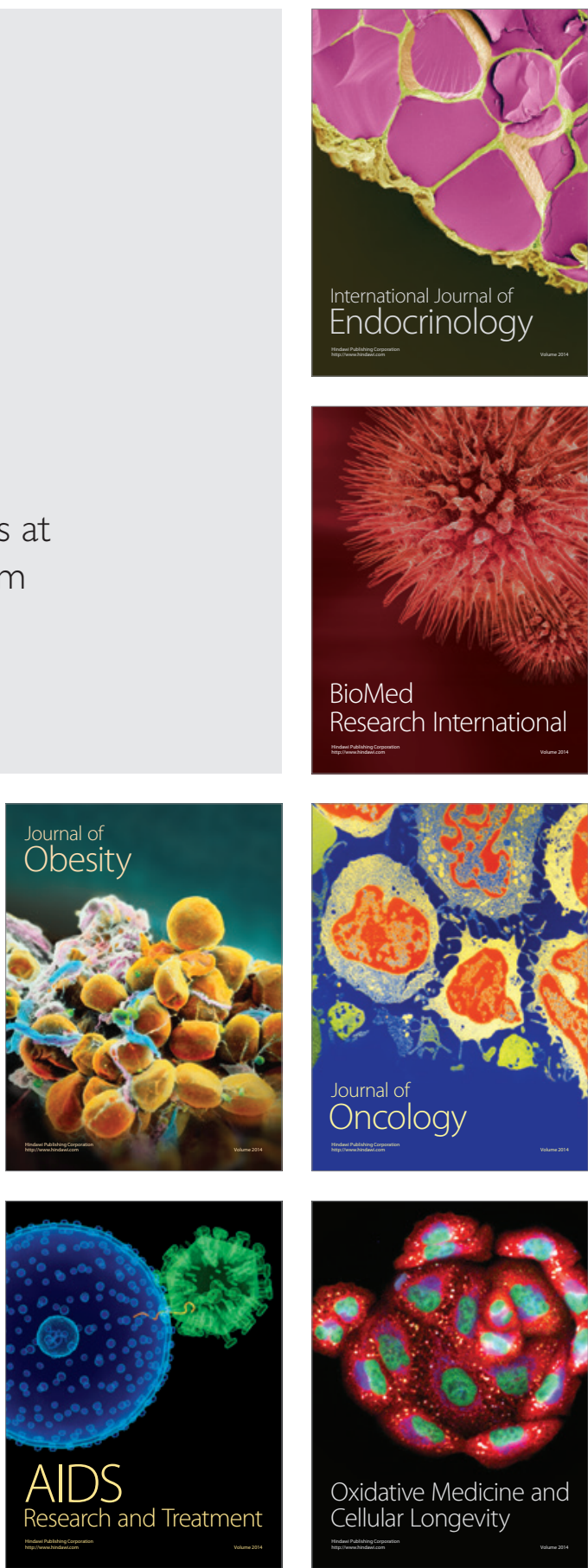